\title{
The transcription factor C-JUN/AP-1 promotes HBV-related liver tumorigenesis in mice
}

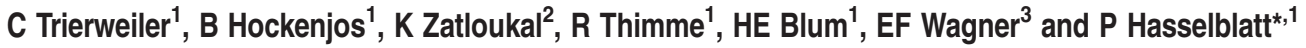

Hepatocellular carcinoma (HCC) develops as a consequence of chronic inflammatory liver diseases such as chronic hepatitis B virus (HBV) infection. The transcription factor c-Jun/activator protein 1 (AP-1) is strongly expressed in response to inflammatory stimuli, promotes hepatocyte survival during acute hepatitis and acts as an oncogene during chemically induced liver carcinogenesis in mice. Here, we therefore aimed to characterize the functions of c-Jun during HBV-related liver tumorigenesis. To this end, transgenic mice expressing all HBV envelope proteins $\left(\mathrm{HBV}^{+}\right)$, an established model of HBV-related HCC, were crossed with knockout mice lacking $c$-Jun specifically in hepatocytes and tumorigenesis was analyzed. Hepatic expression of c-Jun was strongly induced at several time points during tumorigenesis in $\mathrm{HBV}^{+}$mice, whereas expression of other AP-1 components remained unchanged. Importantly, formation of premalignant foci and tumors was strongly reduced in $\mathrm{HBV}^{+}$mice lacking $\mathrm{c}$-Jun. This phenotype correlated with impaired hepatocyte proliferation and increased expression of the cell cycle inhibitor p21, whereas hepatocyte survival was not affected. Progression and prognosis of HBV-related HCC correlates with the expression of the cytokine osteopontin (Opn), an established AP-1 target gene. Opn expression was strongly reduced in $\mathrm{HBV}^{+}$livers and primary mouse hepatocytes lacking $c$-Jun, demonstrating that c-Jun regulates hepatic Opn expression in a cell-autonomous manner. These findings indicate that $\mathrm{c}$-Jun has important functions during HBV-associated tumorigenesis by promoting hepatocyte proliferation as well as progression of dysplasia. Therefore, targeting c-Jun may be a useful strategy to prevent hepatitisassociated tumorigenesis.

Cell Death and Differentiation (2016) 23, 576-582; doi:10.1038/cdd.2015.121; published online 16 October 2015

Hepatocellular carcinoma (HCC) frequently develops as a consequence of chronic inflammatory liver diseases such as chronic hepatitis B virus (HBV) or hepatitis C virus (HCV) infection, chronic inflammation, liver injury, fibrosis and subsequent cirrhosis. HCC is the seventh and fifth most common cancer in women and men, respectively, and its incidence is particularly high in developing countries, in which $\mathrm{HBV}$ and $\mathrm{HCV}$ infections are endemic. ${ }^{1} \mathrm{HBV}$ is considered to be non-cytopathic in most cases and the majority of HBVrelated liver damage has been attributed to hepatic immune responses. ${ }^{2}$ However, HBV may directly promote tumorigenesis by genomic integration of viral DNA or by the action of viral transactivators such as $\mathrm{HBx}$ or mutant preS2 surface proteins. $^{3}$ In keeping with this notion, overexpression of hepatoviral proteins in transgenic mice is sufficient to cause $\mathrm{HCC}$ as shown in $\mathrm{Tg}(\mathrm{A} / \mathrm{b}-1 \mathrm{HB})$ Bri44 mice (hereafter referred to as $\mathrm{HBV}^{+}$mice), which express the large, middle and small isoforms of $\mathrm{HBV}$ surface proteins (LHBs, MHBs and HBs antigens) under the control of the albumin promoter as well as HBx from its endogenous promoter. ${ }^{4}$ Although liver disease in this model does not include viral replication, cccDNA formation and HBV-specific immune cell responses, liver disease in these animals is characterized by accumulation of hepatoviral proteins in the endoplasmic reticulum (ER) and appearance of 'ground glass hepatocytes', liver damage with compensatory proliferation, aneuploidy, dysplasia and eventually HCC, thereby mimicking several aspects of disease pathogenesis in HBV-infected patients. ${ }^{5-7} \mathrm{HBV}^{+}$mice therefore represent a useful tool to genetically dissect molecular pathways related to hepatitis-associated hepatocarcinogenesis. HBV replication and expression of hepatoviral proteins such as HBx strongly activate C-Jun. ${ }^{3,8-10} \mathrm{C}$-Jun is a member of the dimeric activator protein 1 (AP-1) transcription factor family, which mainly consists of Jun (c-Jun, JunB and JunD) and Fos proteins (cFos, FosB, Fra-1 and Fra-2). ${ }^{11}$ c-Jun is expressed as an immediate early gene in response to a variety of stress stimuli, growth factors and subsequent signaling through MAP kinase pathways. It is a major determinant of cell fate in the liver and regulates hepatocyte survival during acute hepatitis and ER stress $^{8,12}$ as well as hepatocyte proliferation following 2/3 partial hepatectomy. ${ }^{13}$ Moreover, c-Jun acts as an oncogene in the liver and strongly promotes liver tumorigenesis in models of chemically induced HCC. ${ }^{14-16}$ However, the functions of C-Jun during HBV-related liver disease are only poorly defined, although the above-mentioned findings strongly suggest that $\mathrm{c}$-Jun may be a central regulator of HBV-mediated hepatocarcinogenesis. To address this issue experimentally, we generated $\mathrm{HBV}^{+}$transgenic mice

${ }^{1}$ Department of Medicine II, University Hospital Freiburg, Hugstetter Strasse 55, D-79106 Freiburg, Germany; ${ }^{2}$ Institute of Pathology, Medical University of Graz, Auenbruggerplatz 25, A-8036 Graz, Austria and ${ }^{3}$ Genes, Development and Disease Group, Cancer Cell Biology Programme, Spanish National Cancer Research Centre, Melchor Fernandez Almagro 3, E-28029 Madrid, Spain

${ }^{*}$ Corresponding author: P Hasselblatt, Department of Medicine II, University Hospital Freiburg, Hugstetter Strasse 55, D-79106 Freiburg, Germany. Tel: +49 76127034010 ; Fax: +49 761270 33530; E-mail: Peter.Hasselblatt@uniklinik-freiburg.de

Abbreviations: HCC, hepatocellular carcinoma; HBV, hepatitis B virus; ER, endoplasmic reticulum; ConA, concanavalin A; ALT, alanine aminotransferase; PMH, primary mouse hepatocytes; AP-1, activator protein 1

Received 16.1.15; revised 21.7.15; accepted 03.8.15; Edited by T Mak; published online 16.10.15 
specifically lacking $c$-Jun in hepatocytes. Our findings indeed indicate that c-Jun has important functions during HBV-related tumorigenesis by regulating hepatocyte proliferation as well as progression of dysplasia.

\section{Results}

Increased expression of c-Jun in $\mathbf{H B V}^{+}$mice. To analyze the expression of c-Jun at various stages of HBV-related tumorigenesis, hepatic expression of c-Jun was determined by quantitative PCR (qPCR) in $\mathrm{HBV}^{+}$mice. In comparison to $\mathrm{HBV}^{-}$controls, $\mathrm{c}$-Jun expression was increased at all time points studied and peaked at around 6 months of age (Figure 1a, Supplementary Figure S1A). Moreover, hepatic C-Jun expression was also increased in a separate infectious mouse model of hepatitis B (Supplementary Figure S1B). In contrast, expression of JunB was not induced in $\mathrm{HBV}^{+}$livers at 6 months of age, whereas JunD expression was slightly increased in $\mathrm{HBV}^{+}$livers irrespective of c-Jun expression (Figure 1b). Expression of Fos genes could not be detected (Figure 1b). Immunohistochemistry of $\mathrm{HBV}^{+}$livers revealed that $c$-Jun was expressed in hepatocytes and inflammatory cells (Figure 1c). Hepatic $c$-Jun expression was substantially reduced in $\mathrm{HBV}^{-} \mathrm{c}_{-} J u n^{\Delta \mathrm{li}}$ and $\mathrm{HBV}^{+} \mathrm{c}_{-} \mathrm{Jun}^{\Delta \mathrm{li}}$ livers (Figure 1b), in which residual c-Jun expression occurred in nonparenchymal cells (Figure 1c). These findings indicate that c-Jun expression is induced following HBV infection and in HBV transgenic mice. Moreover, transgene expression of small and large HBsAg at 6 months of age was analyzed by ELISA and immunoblotting, respectively, and revealed that transgene expression was not altered in the absence of $c$-Jun (Supplementary Figure S1C), indicating that this mouse model was indeed suitable to further study the functions of c-Jun in HBV-related tumorigenesis.
c-Jun strongly promotes HBV-related tumorigenesis. To determine the impact of c-Jun expression on HBV-related tumorigenesis, $\mathrm{HBV}^{+} c-J u n^{\mathrm{f} / \mathrm{f}}$ and $\mathrm{HBV}^{+} c-J u n^{\Delta \mathrm{li}}$ mice were analyzed at 12 months of age. No obvious macroscopical liver abnormalities were observed in $H_{B} V^{-} \quad c-J u n^{f / f}$ and $c-J u n^{\Delta l i}$ mice (Figure 2a). In contrast, liver tumors were apparent macroscopically in $15 / 22 H_{B V^{+}} C$-Jun ${ }^{\mathrm{f} / \mathrm{f}}$ mice, whereas livers appeared normal and tumor-free in all 16 $\mathrm{HBV}^{+} \mathrm{C}$-Jun ${ }^{\Delta \mathrm{li}}$ mice (Figure 2a). These findings correlated with a significant increase in liver weight in $\mathrm{HBV}^{+} \mathrm{C}-\mathrm{Jun}{ }^{\mathrm{f} / \mathrm{fi}}$ as compared with $\mathrm{HBV}^{+} \mathrm{c}$-Jun ${ }^{\Delta \mathrm{li}}$ mice (Figure $2 \mathrm{~b}$ ). Moreover, tumorigenesis was also quantified microscopically and confirmed that tumorigenesis was strikingly reduced at this time point in $\mathrm{HBV}^{+}$mice lacking $c$-Jun (Figure 2c). Histopathological hallmarks of $\mathrm{HBV}^{+}$livers include the appearance of ground glass hepatocytes due to accumulation of surface proteins in the ER, inflammation, cellular atypia and the appearance of atypical foci, which progress to hyperplastic nodules, adenoma, and eventually $\mathrm{HCC}^{5}$ Infiltration of $\mathrm{CD}^{+}$ T cells and $\mathrm{Ly}_{6 \mathrm{G}}{ }^{+}$neutrophils was not altered in $\mathrm{HBV}^{+}$mice lacking $c$-Jun (Supplementary Figure S2A). The appearance of ground glass hepatocytes, inflammation and cellular atypia were comparable in $\mathrm{HBV}^{+} \mathrm{c}$-Jun ${ }^{\mathrm{f} / \mathrm{f}}$ and $\mathrm{HBV}^{+} \mathrm{c}$-Jun ${ }^{\Delta \mathrm{li}}$ livers as determined by histopathological analysis, whereas numbers of atypical foci and subsequent pro-tumorigenic alterations were profoundly reduced in the absence of $c$-Jun (Figures $3 \mathrm{a}$ and b). c-Jun expression was observed in most HBV-related pathologies including ground glass hepatocytes, adenoma and HCC (Supplementary Figure S2B). It should be noted that most of the tumor lesions observed in $\mathrm{HBV}^{+}$livers here were hyperplastic nodules or adenomas and that the prevalence of $\mathrm{HCC}$ in $\mathrm{HBV}^{+} \mathrm{c}-J u n^{\mathrm{f} / \mathrm{f}}$ mice was low at the age of 12 months. However, it has been shown that the precursor lesions observed here can be used as tumor surrogate and a

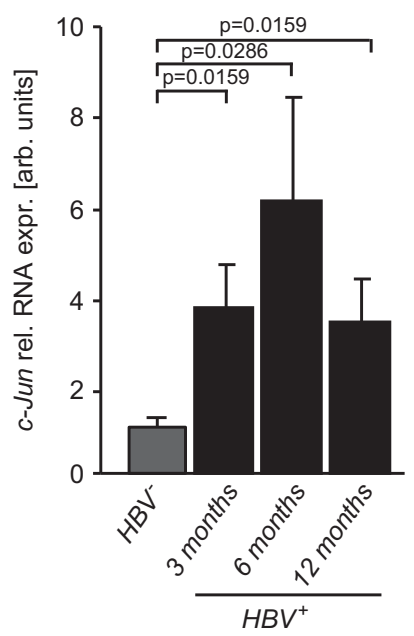

b

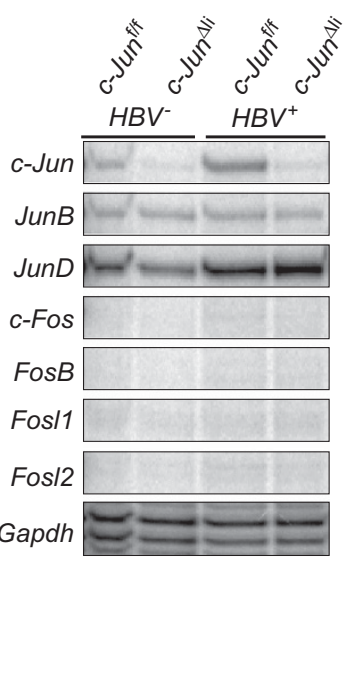

c

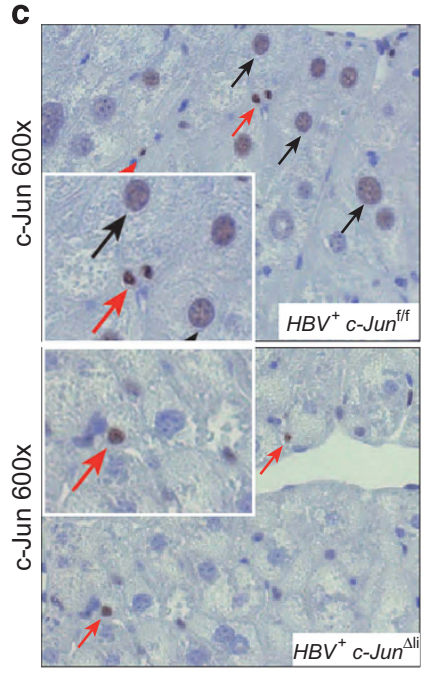

Figure 1 c-Jun expression is induced in $\mathrm{HBV}^{+}$mice. (a) Hepatic c-Jun mRNA expression at the indicated time points was determined by qPCR and normalized to the expression in HBV livers ( $n \geq 4$ per genotype and time point, significance was tested by Mann-Whitney test). (b) Hepatic expression of AP- 1 family members in mice with the indicated genotypes at 6 months of age was analyzed by RNase protection assay. Gapdh expression was used as loading control. (c) c-Jun expression in livers of 6-month-old mice with the indicated genotypes as determined by immunohistochemistry. Note the expression in hepatocytes (black arrows) and inflammatory cells (red arrows) 
a

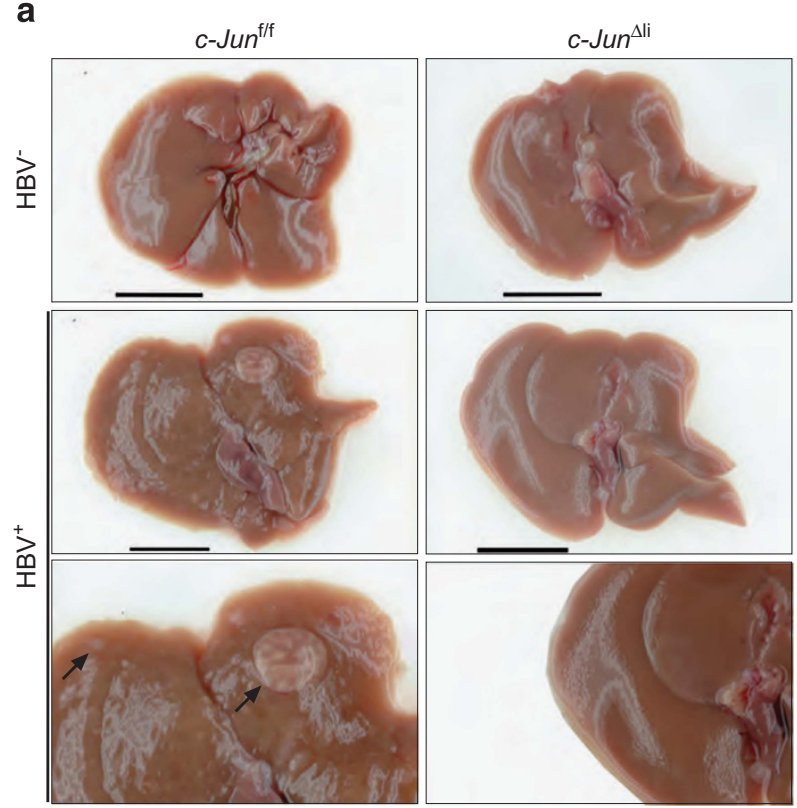

b
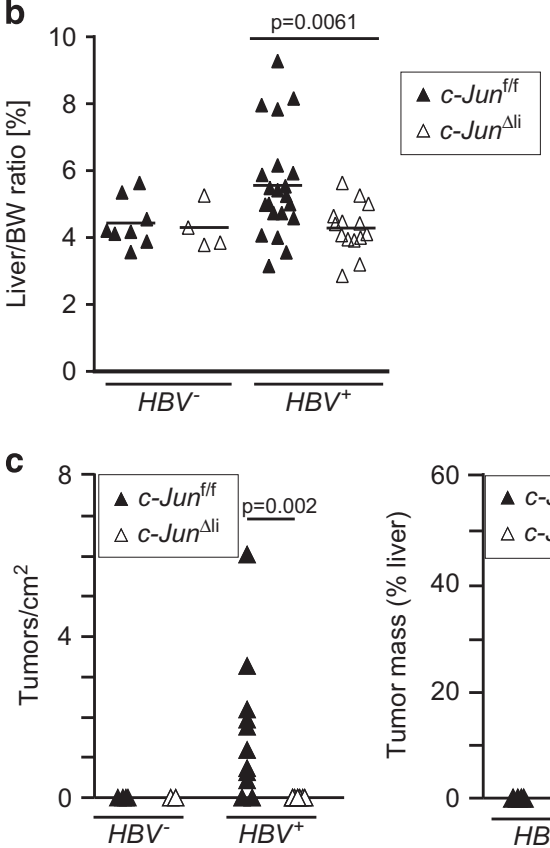

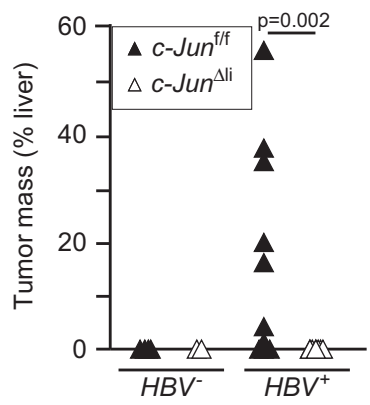

Figure 2 Liver tumorigenesis is strongly reduced in 12-month-old $\mathrm{HBV}^{+}$mice lacking c-Jun. (a) Macroscopical images of representative livers of mice with the indicated genotypes. Black bar $=1 \mathrm{~cm}$. (b) Liver weight was determined as liver/body weight ratio, ( $n=4-21$ per genotype, significance was tested by Mann-Whitney test (MWT)). (c) Liver tumorigenesis was quantified histologically and is given as tumor numbers per $\mathrm{cm}^{2}$ liver tissue. Tumor load was determined as a ratio of tumorous tissue and non-tumorous tissue and is given in (\%). Significance was tested by MWT

predict hepatocarcinogenesis at later time points. ${ }^{7}$ Moreover, expression of $\mathrm{HBsAg}$ is gradually downregulated during neoplastic transformation and areas lacking transgene expression can therefore be used as a predictor of tumorigenesis. ${ }^{7}$ Although transgene expression was comparable at 6 months of age, immunohistochemistry revealed that $\mathrm{HBsAg}$ expression was lost in many liver areas of $\mathrm{HBV}^{+}$ $C-J u n^{\mathrm{f} / \mathrm{f}}$ mice at 12 months of age consistent with the formation of preneoplastic foci, but rarely in $\mathrm{HBV}^{+} \mathrm{C}-\mathrm{Jun}^{\mathrm{Ali}}$ mice (Supplementary Figure S1D). Tumor analysis was therefore not extended beyond 12 months. In conclusion, our findings indicate that $\mathrm{C}$-Jun is an important regulator of tumorigenesis in $\mathrm{HBV}^{+}$mice.

c-Jun promotes hepatocyte proliferation in $\mathrm{HBV}^{+}$livers. c-Jun is an important regulator of hepatocyte proliferation, survival and related stress responses. We have previously shown that c-Jun expression determines hepatocyte survival during T-cell-mediated hepatitis as well as during chemically induced ER stress, ${ }^{8,12}$ raising the question whether similar functions may be relevant in $\mathrm{HBV}^{+}$mice. However, expression of the established ER stress marker genes BiP and Gadd153 as well as $X B P-1$ splicing were not significantly induced in $\mathrm{HBV}^{+}$as compared with $\mathrm{HBV}$ livers and not altered in the absence of $c$-Jun (Supplementary Figure S3A). To further analyze the impact of c-Jun expression on hepatocyte damage, serum transaminase concentrations were measured. Serum alanine aminotransferase (ALT) concentrations in $\mathrm{HBV}^{+}$mice peaked at the age of 6 months and decreased thereafter, but were not altered in the absence of $c$-Jun (Figure 4a), suggesting that c-Jun is not involved in regulating hepatocyte damage or survival in this model. In keeping with this notion, no difference in cell death could be observed by TUNEL staining, whereas the numbers of $\mathrm{TUNEL}^{+}$cells were very low overall (Supplementary Figure S4). Previous findings suggest that C-Jun promotes DEN-mediated and HCV-related hepatocarcinogenesis by regulating the expression of Birc5 or activation of Stat3. ${ }^{15,16}$ In $\mathrm{HBV}^{+}$mice, hepatic Birc5 expression was not altered in the absence of $c$-Jun (Supplementary Figure S3A). Although Stat3 phosphorylation was evident in $\mathrm{HBV}^{+}$livers, its phosphorylation as well as expression of Socs3 was not changed in $c$-Jun-deficient livers (Supplementary Figure $\mathrm{S} 3 \mathrm{~B}$ ), indicating that the Stat3 pathway does not contribute to the differences observed in $H B V^{+} c-J u n^{-1 i}$ mice.

Hepatocarcinogenesis in $\mathrm{HBV}^{+}$mice is preceded by sustained hepatocyte proliferation. ${ }^{17}$ Hepatocyte proliferation was significantly reduced in $\mathrm{HBV}^{+}$livers lacking $\mathrm{c}$-Jun at the age of 6 and 12 months (Figures $4 b$ and $c$ ), whereas hepatic expression of the cell cycle inhibitor and tumor suppressor p21 was increased (Figure 4c; Supplementary Figure S5A). Moreover, $\mathrm{HBV}^{+} \mathrm{c}-\mathrm{Jun} \mathrm{n}^{\mathrm{f} / \mathrm{f}}$ primary mouse hepatocytes $(\mathrm{PMH})$ were isolated and infected with adenoviral vectors expressing Cre recombinase, which resulted in profound inhibition of c-Jun expression, whereas p21 expression was significantly induced (Supplementary Figure S5B). These findings strongly suggest that c-Jun interferes with hepatic p21 expression in a cell-autonomous manner.

c-Jun regulates hepatic Opn expression. Our finding that progression of dysplasia was reduced in $c$-Jun mutant livers raises the question whether $c$-Jun also regulates the 

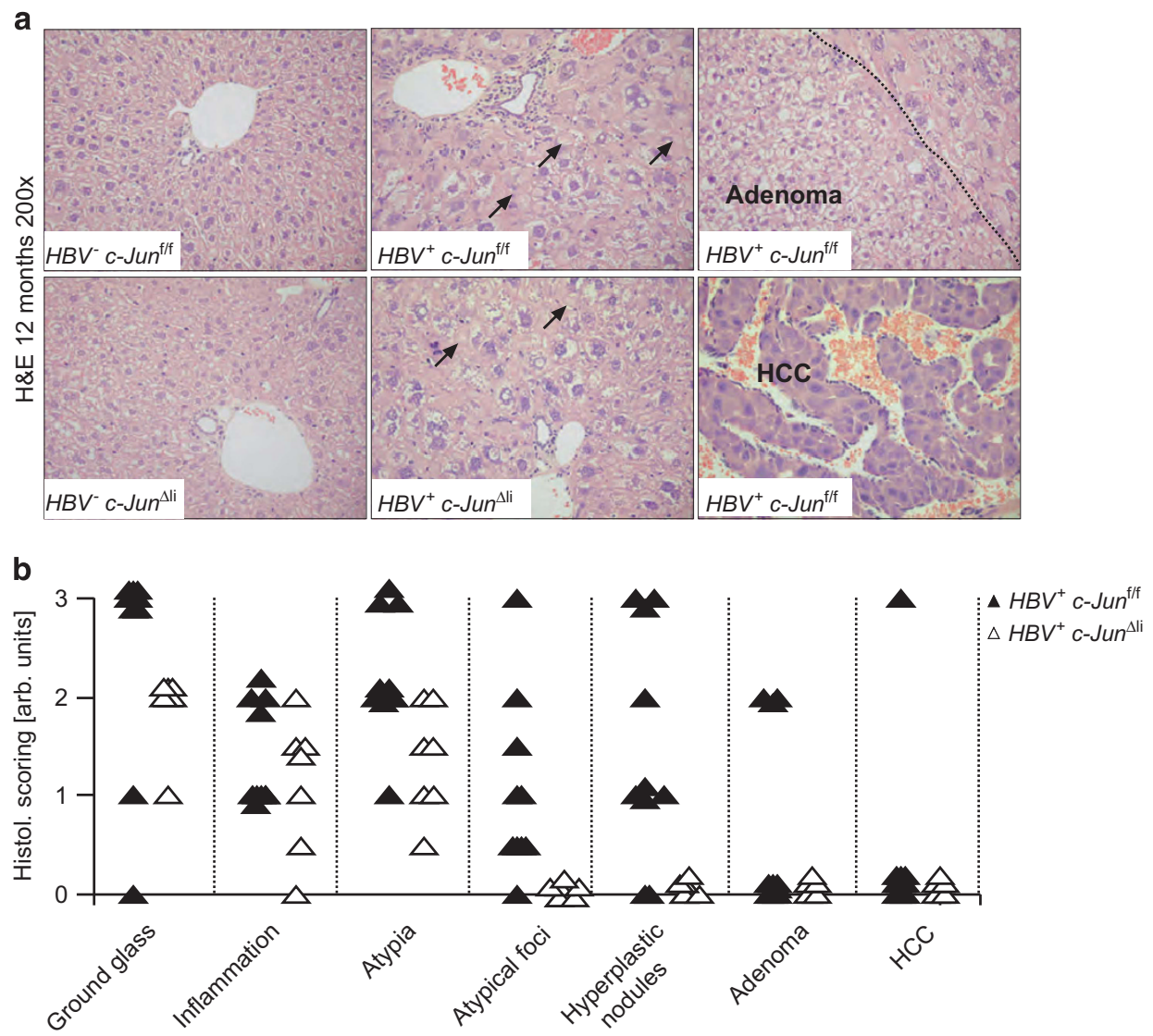

Figure 3 Absence of atypical foci and dysplastic progression in $\mathrm{HBV}^{+}$mice lacking $\mathrm{c}$-Jun. (a) Representative histological stainings of mice with the indicated genotypes are shown (12 months of age). Note the appearance of ground glass hepatocytes in $\mathrm{HBV}^{+}$mice, which occurred irrespective of c-Jun expression. (b) Histological alterations observed in mice with the indicated genotypes were scored using an arbitrary scale from 0 to 3

expression of genes related to the progression of dysplastic lesions such as osteopontin (Opn). Opn is highly expressed in $\mathrm{HCC}$ and correlates with histological tumor grading, tumor stage as well as early recurrence following surgical resection, in particular in HBV-infected patients. ${ }^{18}$ Importantly, hepatic $\mathrm{Opn}$ expression was induced in $\mathrm{HBV}^{+}$mice at the age of 12 months as determined by qPCR, ELISA and immunoblotting, but reduced in the absence of $c$-Jun (Figures $5 \mathrm{a}$ and b; Supplementary Figure S3B). To address the question whether c-Jun promotes hepatocellular Opn expression in a cell-autonomous manner, $\mathrm{PMH}$ were isolated. Opn expression was indeed reduced in $\mathrm{HBV}^{+} c-J u n^{\Delta \mathrm{i}} \mathrm{PMH}$ as determined by qPCR and ELISA (Figure $5 \mathrm{c}$ ). Opn expression was also impaired in $\mathrm{HBV}^{+} \mathrm{C}-\mathrm{Jun} \mathrm{h}^{\mathrm{t/f}} \mathrm{PMH}$ upon adenoviral transfer of Cre recombinase (Supplementary Figure S5B), indicating that this phenotype was independent of the AlfpCre transgene used here. Immunohistochemistry further revealed that Opn expression was restricted to bile duct epithelia in $\mathrm{HBV}^{+}{ }_{c}$-Jun ${ }^{\Delta l i}$ livers. In $\mathrm{HBV}^{+} \mathrm{c}_{\text {-Jun }}^{\mathrm{f} / \mathrm{f}}$ livers, it occurred in some dysplastic foci and scattered hepatocytes as well as in bile duct epithelia and proliferative ductular cells. These cells also expressed Sox9 and cytokeratin 19 (CK19) (Figure 5d), which also identify hepatic and liver cancer progenitor cells. ${ }^{19}$ Moreover, hepatic Opn expression was also reduced in $\mathrm{C}$-Jun ${ }^{\Delta \mathrm{li}}$ mice during acute concanavalin A
(ConA)-mediated hepatitis (Supplementary Figure S5C), indicating that C-Jun regulates Opn expression also in other models of inflammatory liver disease. In conclusion, these findings indicate that C-Jun regulates hepatic Opn expression in a cell-autonomous manner, which in turn promotes HBVrelated tumorigenesis.

\section{Discussion}

Expression of the AP-1 transcription factor C-Jun has been observed in many molecular subtypes of human $\mathrm{HCC}^{20}$ and is particularly increased in HBV-related HCC. ${ }^{21}$ c-Jun strongly promotes liver tumorigenesis in mouse models of chemically induced $\mathrm{HCC}^{14-16}$ and also determines hepatocyte fate in a variety of inflammatory stress conditions including T-cellmediated hepatitis and ER stress, ${ }^{8,12}$ suggesting that C-Jun may also contribute to the pathogenesis of hepatitisassociated HCC. Here, we demonstrate that c-Jun strongly promotes liver tumorigenesis in $\mathrm{HBV}^{+}$mice, a mouse model that mimics many aspects of the series of pathophysiological events leading to HCC in patients with chronic HBV infection. ${ }^{5,17}$ C-Jun regulates hepatocyte survival following DEN-mediated tumor initiation by interfering with c-Fos and Birc5 ${ }^{15}$ or with Nos2 and Stat3 signaling in DEN-treated HCV transgenic mice ${ }^{16}$ Interestingly, no differences in liver damage 

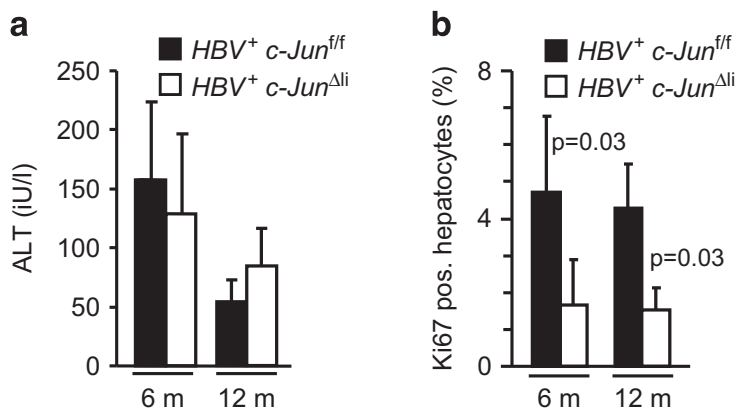

C
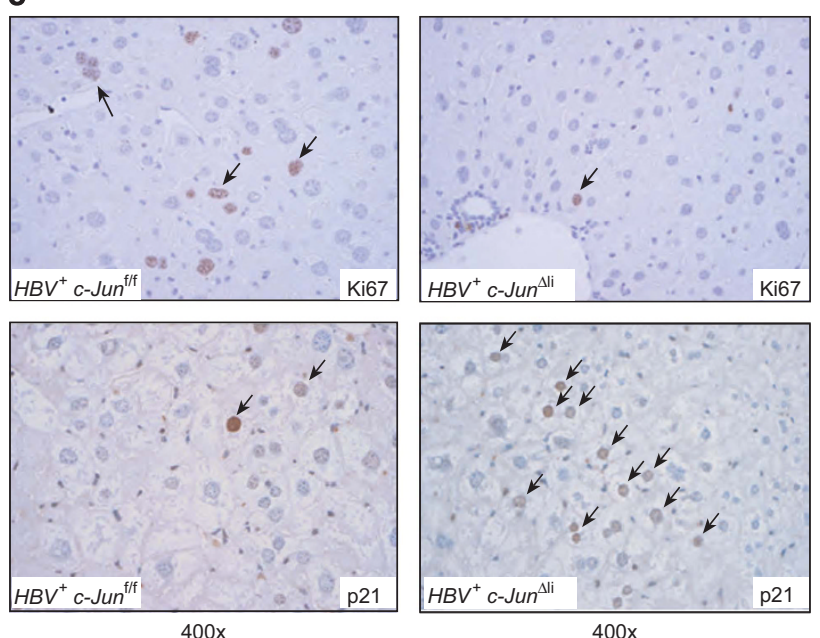

Figure 4 c-Jun promotes hepatocyte proliferation by interfering with p21. (a) Liver damage in mice with the indicated genotypes was determined by analysis of serum ALT concentrations at 6 and 12 months, respectively. ( $n=12-16$ per genotype and time point). ( $b$ and $\mathbf{c}$ ) Hepatocyte proliferation was assessed by immunohistochemistry for Ki67 at 6 and 12 months of age, respectively. For quantification, three representative high-power fields were analyzed per mouse. ( $n \geq 4$ per genotype and time point; significance was tested by Mann-Whitney test). p21 expression was analyzed by immunohistochemistry

could be detected in $\mathrm{HBV}^{+}$mice lacking $\mathrm{c}$-Jun, in which Birc5 and Stat3 expression were also not altered. In contrast, hepatocyte proliferation was reduced in $\mathrm{HBV}^{+}$mice lacking c-Jun, indicating that c-Jun rather regulates hepatocyte proliferation in these mice. Increased hepatocyte proliferation may occur in response to HBV-mediated liver damage or through the hepatoviral transactivator $\mathrm{HBx}$ or truncated preS2 proteins, all of which are expressed in $\mathrm{HBV}^{+}$mice and have been shown to promote C-Jun expression. ${ }^{22,23}$ Impaired hepatocyte proliferation in the absence of $c$-Jun correlated with increased expression of the cell cycle inhibitor p21, which is consistent with previous findings in $c-J_{u n}{ }^{-1-}$ mouse embryonic fibroblasts and in $c$-Jun ${ }^{\Delta l i}$ mice following 2/3 partial hepatectomy. ${ }^{13,24}$

Expression of the cytokine Opn is an early predictor of $\mathrm{HCC}$ formation and predicts its recurrence upon surgical resection, in particular in HBV-infected patients. ${ }^{18,25}$ Interestingly, hepatic Opn expression was reduced in the absence of $c$-Jun in a cell-autonomous manner. Opn is an established AP-1 target gene and its expression is likely regulated by c-Jun and the Fos proteins Fra- 1 or Fra-2. ${ }^{26-28}$ In $\mathrm{HBV}^{+}$mice, Opn expression occurred predominantly in bile duct epithelia, proliferating ductular cells and hepatocytes. Recent findings indicate that Opn promotes proliferative ductular reactions following liver damage. ${ }^{29}$ Interestingly, this ductular reaction and cell numbers expressing the progenitor markers Opn, Sox9 and CK19 were reduced in $\mathrm{HBV}^{+}$mice lacking $c$-Jun. These findings suggest that c-Jun may be involved in regulating progenitor cell responses during HBV-related liver damage and hepatocarcinogenesis, which is consistent with the clinical observation that AP-1 expression identifies a subgroup of liver progenitor cell-derived tumors with poor prognosis. $^{30}$

In conclusion, our findings indicate that c-Jun has important functions during HBV-related hepatocarcinogenesis. Interactions between c-Jun and p21 very likely promote compensatory hepatocyte proliferation, which is a prerequisite for HBVrelated hepatocarcinogenesis. Moreover, our findings suggest that c-Jun regulates hepatic Opn expression, thereby contributing to the progression of dysplasia, possibly by interfering with progenitor cell responses. Targeting of c-Jun may therefore be a reasonable approach to interfere with the formation of hepatitis-associated HCC.

\section{Materials and Methods}

Mice. Mice with conditional alleles of c-Jun $\left(c-J u n^{f / f}\right)$ were crossed with transgenic AlfpCre mice to obtain animals with hepatocyte-specific knockout of $c$-Jun $\left(c-J u n^{\Delta l i}\right){ }^{31}$ These mice were crossed with $\mathrm{HBV}^{+}$mice $(\mathrm{Tg}(\mathrm{Alb}-1 \mathrm{HBV}) \mathrm{Bri} 44){ }^{4}$ Animals were bred on a mixed genetic background (C57BL/6 $\times 129 / \mathrm{Sv} \times \mathrm{FVB} / \mathrm{N}$ ) and housed under specific pathogen-free conditions. Either $c$-Jun $n^{f / 4}$ or AlfpCre $c-J u n^{t / f}$ littermates lacking the HBV transgene (HBV) were used as controls. All animals received humane care and experiments were performed in accordance with local and institutional regulations. In some animals, acute T-cell-mediated hepatitis was induced by injection of ConA $(10 \mathrm{mg} / \mathrm{kg}$ body weight, Sigma, Taufkirchen, Germany) as previously described. ${ }^{12}$ Isolation of PMH was performed as described. ${ }^{8}$ In some experiments, PMH were infected by adenoviral vectors expressing GFP or Cre recombinase (Gene Transfer Vector Core Facility, University of lowa, lowa, IA, USA). Briefly, PMH were incubated with adenovirus (10 MOI) for $3 \mathrm{~h}$ and collected after $48 \mathrm{~h}$. To study c-Jun expression in a mouse model of HBV infection, C57BL/6 mice were infected with adenovirus ( $10^{9} \mathrm{U}$ per mouse) encoding an 1.3 HBV overlength genome (AdenoHBV) or empty control virus as described. ${ }^{32}$ Mice were analyzed 7 days after adenoviral infection.

Cytotoxicity assays and ELISA. Liver damage was determined by analysis of serum ALT using semi-automated clinical routine methods. Opn or LHB concentrations in liver lysates, serum and cell culture supernatants were determined by ELISA according to the manufacturer's protocol (DY441, R\&D Systems, Wiesbaden, Germany and EL10018, Abazyme, Needham, MA, USA).

Histology and Immunohistochemistry. For histology, livers were fixed in $3.7 \%$ neutral buffered formaldehyde at $4^{\circ} \mathrm{C}$ and embedded in paraffin. Immunohistochemistry was performed using the Envision kit (Dako, Hamburg, Germany) and antibodies for c-Jun (\#9165; Cell Signaling, NEB, Frankfurt, Germany), Ki67 (\#301119; Novocastra, Newcastle, UK), p21 (Santa Cruz Biotechnologies, Santa Cruz, CA, USA), Opn (\#AF808; R\&D Systems), HBsAg (HB100; BioLogo, Kronshagen, Germany), Sox9 (AB5535; Millipore, Schwalbach, Germany), CK19 (TROMA, kindly provided by R. Kemler), CD3 (clone SP7; Thermo Scientific, Fremont, CA, USA) or Ly6G (\#551459; BD, Heidelberg, Germany) were used.

qPCR and RNA protection analysis. For isolation of RNA, macrodissected liver tissue was snap-frozen in liquid nitrogen and total RNA was isolated using Qiazol (Qiagen, Hilden, Germany). For isolation of total RNA from PMH, cells were lysed on their culture plate and RNA was extracted using the RNeasy kit (Qiagen). Complementary DNA synthesis was performed using the First Strand cDNA synthesis kit (Fermentas, St. Leon-Rot, Germany). qPCR was performed with SYBR Green (Invitrogen, Karlsruhe, Germany) and 10\% dimethylsulfoxide 

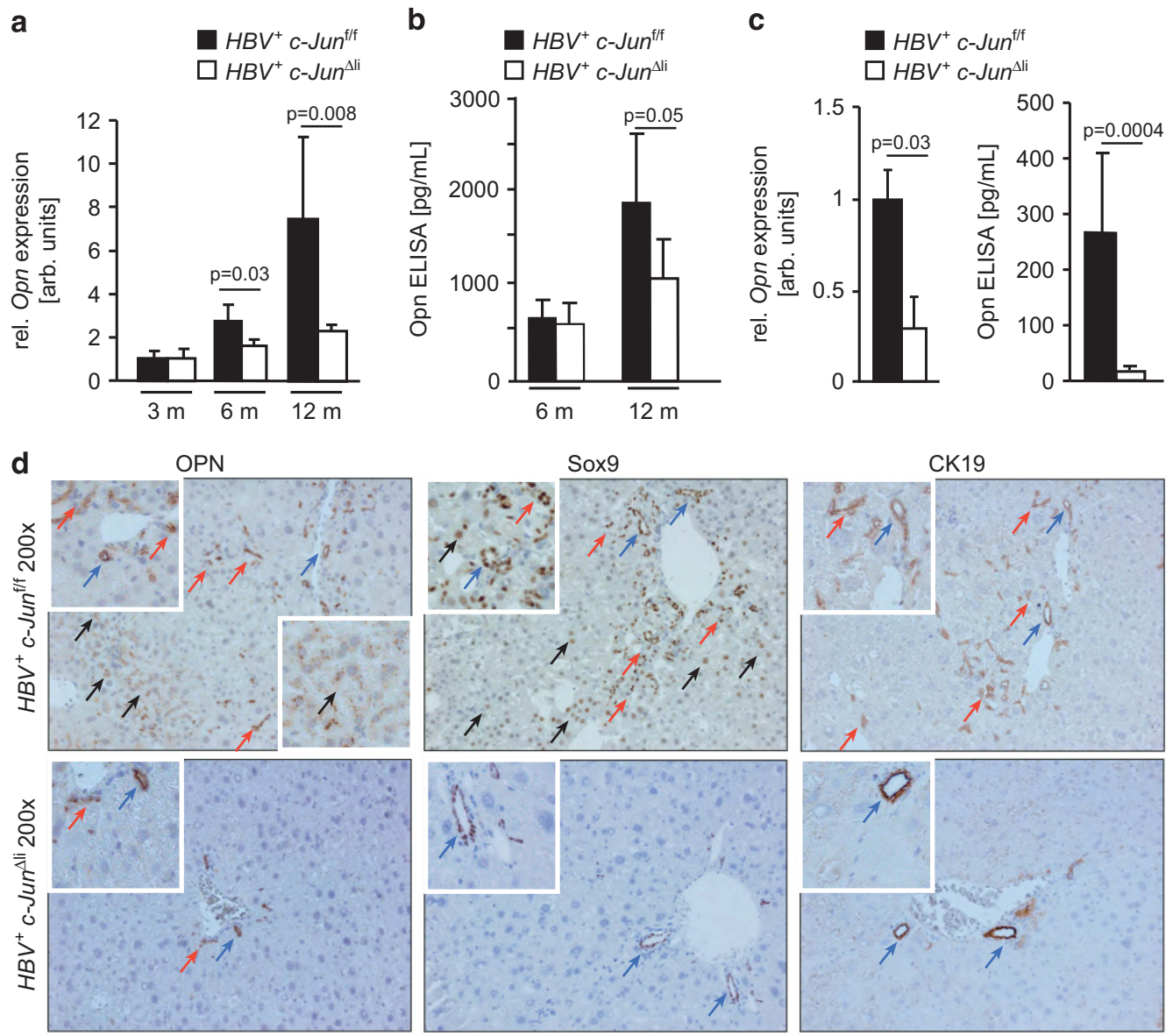

Figure 5 c-Jun regulates hepatic Opn expression. (a and $\mathbf{b})$ Hepatic Opn expression was determined at the indicated time points by qPCR (a) and ELISA (b), respectively. Opn RNA expression was normalized to expression in HBV controls. ( $n \geq 4$ per genotype and time point, significance was tested by Mann-Whitney test (MWT)). (c) Opn expression in primary hepatocytes was determined by qPCR and ELISA from the supernatant, respectively. ( $n \geq 4$ per genotype, significance was tested by MWT). (d) Expression analysis of Opn, Sox9 and CK19 by immunohistochemistry. Hepatocytes, bile duct epithelia and proliferative ductular cells are indicated by black, blue and red arrows, respectively

(Sigma) on a 480 Lightcycler (Roche, Mannheim, Germany; 40 cycles: $30^{\prime \prime} 95^{\circ} \mathrm{C}$; $30^{\prime \prime} 60^{\circ} \mathrm{C} ; 40^{\prime \prime} 72^{\circ} \mathrm{C}$ ). Loading was normalized to Hprt and Actin messenger RNA and $18 \mathrm{~S}$ ribosomal RNA. All primers were designed using Primer-BLAST (www. ncbi.nlm.nih.gov/tools/primer-blast). Primers were synthesized by Microsynth, Balgach, Switzerland, and the specificity of the PCR products was analyzed by melting curve analysis.

Primer sequences were as follows: Actin: 5'-AGCCATGTACGTAGCCATCC-3'; 5'-CTCTCAGCTGTGGTGGTGAA-3'; $18 \mathrm{~S}$ : 5'-CGCGGTTCTATTTTGTTGGT-3'; 5'-AGTCGGCATCGTTTATGGTC-3'; Hprt: 5'-GCTTGCTGGTGAAAAGGACCTC-3'; 5'-CAAATCAAAAGTCTGGGGACGC-3' c-Jun: 5'-AAAACCTTGAAAGCGCAAAA-3'; 5'-CGCAACCAGTCAAGTTCTCA'; Opn: 5'-TGCACCCAGATCCTATAGCC-3'; 5'-CTCC ATCGTCATCATCATCG-3' BiP: 5'-AGTGGTGGCCACTAATGGAG-3'; 5'-CAATCC TTGCTTGATGCTGA-3' Gadd153: 5'-GCATGAAGGAGAAGGAGCAG-3'; 5'-CTTCC GGAGAGACAGACAGG-3' spliced Xbp-1: 5'-GAGTCCGCAGCAGGTG-3'; 5'-GTGT CAGAGTCCATGGGA-3' BirC5: 5'-CTCTCCCGGCATGCTCTGCG-3'; 5'-CCGCC ATTCGCTCTGGGGTG-3' p21: 5'-AACTACCAGCTGTGGGGTGA-3'; 5'-GACAAC GGCACACTTTGCTC-3'; Sesn2. 5'-TAGCCTGCAGCCTCACCTAT-3'; 5'-GATTITGA GGTTCCGTTCCA-3'; Ho-1: 5'-CACGCATATACCCGCTACCT-3'; 5'-CCAGAGTGT TCATTCGAGCA-3'; Att-3: 5'-CTAGAATCCCAGCAGCCAAG-3'; 5'-GGCCAGCTAG GTCATCTGAG-3'; RNase protection analyses were performed using the Riboquant multiprobe RPA system mFos/Jun (BD).

Western blot analysis. Hepatocyte and total liver lysates were analyzed by immunoblot using antibodies for c-Jun (\#9165; Cell Signaling), phospho-c-Jun (Ser63, \#9261; Cell Signaling), $\beta$-Actin (\#A2066; Sigma), phospho-Stat3 (Tyr705, \#9131; Cell Signaling), Stat3 (\#06-596; Millipore), Socs3 (\#2932; Cell
Signaling), p21 (Santa Cruz), Opn (\#AF808; R\&D Systems) or LHBs (MA18/07; kindly provided by M Nassal).

Statistics. Data in bar graphs represent mean \pm S.D. Normal distribution of the data was tested using Graphpad PRISM software (La Jolla, CA, USA). Statistical analysis was performed using the nonparametric Mann-Whitney test or nondirectional two-tailed Student's $t$-test as indicated in the figure legends.

\section{Conflict of Interest}

The authors declare no conflict of interest.

Acknowledgements. The authors thank $\mathrm{M}$ Geissler for providing $\mathrm{HBV}^{+}$mice, $R$ Kemler and $M$ Nassal for providing antibodies, U Protzer for providing liver samples of adenoHBV-infected mice, O Sansom for help with p21 immunohistochemistry, the animal house staff at the Institute of Molecular Pathology in Vienna, and at Freiburg University Hospital for support, S MacNelly for isolating primary mouse hepatocytes and $L$ Bakiri and $R$ Eferl for helpful comments. This work was supported by the German Research Foundation (DFG, www.dfg.de) grants Ha4314/2-1 and Ha4314/22. E.F.W. is supported by a grant from the Spanish Ministry of Economy (BFU201240230) and an ERC-Advanced grant (ERC-FCK/2008/37).

\section{Author contributions}

EFW, HEB and PH designed the study; CT, BH and PH performed experiments; CT, $\mathrm{KZ}, \mathrm{RT}$ and $\mathrm{PH}$ analyzed the data and $\mathrm{PH}$ wrote the paper. 
1. El-Serag HB. Epidemiology of viral hepatitis and hepatocellular carcinoma. Gastroenterology 2012; 142: 1264-1273.e1.

2. Guidotti LG, Chisari FV. Immunobiology and pathogenesis of viral hepatitis. Annu Rev Pathol 2006; 1: 23-61.

3. Arzumanyan A, Reis HM, Feitelson MA. Pathogenic mechanisms in HBV- and HCV-associated hepatocellular carcinoma. Nat Rev Cancer 2013; 13: 123-135.

4. Chisari FV, Klopchin K, Moriyama T, Pasquinelli C, Dunsford HA, Sell S et al. Molecular pathogenesis of hepatocellular carcinoma in hepatitis B virus transgenic mice. Cell 1989; 59: 1145-1156.

5. Li Y, Tang ZY, Hou JX. Hepatocellular carcinoma: insight from animal models. Nat Rev Gastroenterol Hepatol 2011; 9: 32-43.

6. Bakiri L, Wagner EF. Mouse models for liver cancer. Mol Oncol 2013; 7: 206-223.

7. Toshkov I, Chisari FV, Bannasch P. Hepatic preneoplasia in hepatitis B virus transgenic mice. Hepatology 1994; 20: 1162-1172.

8. Fuest M, Willim K, Macnelly S, Fellner N, Resch GP, Blum HE et al. The transcription factor c-Jun protects against sustained hepatic endoplasmic reticulum stress thereby promoting hepatocyte survival. Hepatology 2012; 55: 408-418.

9. Twu JS, Lai MY, Chen DS, Robinson WS. Activation of protooncogene c-jun by the X protein of hepatitis B virus. Virology 1993; 192: 346-350.

10. Benn J, Su F, Doria M, Schneider RJ. Hepatitis B virus HBx protein induces transcription factor AP-1 by activation of extracellular signal-regulated and c-Jun $\mathrm{N}$-terminal mitogenactivated protein kinases. J Virol 1996; 70: 4978-4985.

11. Eferl R, Wagner EF. AP-1: a double-edged sword in tumorigenesis. Nat Rev Cancer 2003; 3 : 859-868.

12. Hasselblatt $P$, Rath $M$, Komnenovic V, Zatloukal $K$, Wagner EF. Hepatocyte survival in acute hepatitis is due to c-Jun/AP-1-dependent expression of inducible nitric oxide synthase. Proc Natl Acad Sci USA 2007; 104: 17105-17110.

13. Stepniak E, Ricci R, Eferl R, Sumara G, Sumara I, Rath M et al. c-Jun/AP-1 controls liver regeneration by repressing p53/p21 and p38 MAPK activity. Genes Dev 2006; 20: 2306-2314.

14. Eferl R, Ricci R, Kenner L, Zenz R, David JP, Rath M et al. Liver tumor development. c-Jun antagonizes the proapoptotic activity of p53. Cell 2003; 112: 181-192.

15. Min L, Ji Y, Bakiri L, Qiu Z, Cen J, Chen X et al. Liver cancer initiation is controlled by AP-1 through SIRT6-dependent inhibition of survivin. Nat Cell Biol 2012; 14: 1203-1211.

16. Machida K, Tsukamoto H, Liu JC, Han YP, Govindarajan S, Lai MM et al. c-Jun mediates hepatitis $C$ virus hepatocarcinogenesis through signal transducer and activator of transcription 3 and nitric oxide-dependent impairment of oxidative DNA repair. Hepatology 2010; 52: 480-492.

17. Huang SN, Chisari FV. Strong, sustained hepatocellular proliferation precedes hepatocarcinogenesis in hepatitis B surface antigen transgenic mice. Hepatology 1995; 21: 620-626.

18. Shang S, Plymoth A, Ge S, Feng Z, Rosen HR, Sangrajrang S et al. Identification of osteopontin as a novel marker for early hepatocellular carcinoma. Hepatology 2012; 55: 483-490.
19. He G, Dhar D, Nakagawa $H$, Font-Burgada J, Ogata $H$, Jiang $Y$ et al. Identification of liver cancer progenitors whose malignant progression depends on autocrine IL-6 signaling. Cell 2013; 155: 384-396.

20. Malz M, Pinna F, Schirmacher P, Breuhahn K. Transcriptional regulators in hepatocarcinogenesis-key integrators of malignant transformation. J Hepatol 2012; 57: 186-195.

21. Guo L, Guo Y, Xiao S, Shi X. Protein kinase p-JNK is correlated with the activation of AP-1 and its associated Jun family proteins in hepatocellular carcinoma. Life Sci 2005; 77 : 1869-1878.

22. Tanaka $Y$, Kanai F, Ichimura T, Tateishi K, Asaoka Y, Guleng B et al. The hepatitis B virus $X$ protein enhances AP-1 activation through interaction with Jab1. Oncogene 2006; 25: 633-642.

23. Hildt E, Munz B, Saher G, Reifenberg K, Hofschneider PH. The PreS2 activator MHBs $(\mathrm{t})$ of hepatitis $B$ virus activates $c$-raf-1/Erk2 signaling in transgenic mice. EMBO J 2002; 21: 525-535.

24. Schreiber M, Kolbus A, Piu F, Szabowski A, Mohle-Steinlein U, Tian J et al. Control of cell cycle progression by c-Jun is p53 dependent. Genes Dev 1999; 13: 607-619.

25. Yu MC, Lee YS, Lin SE, Wu HY, Chen TC, Lee WC et al. Recurrence and poor prognosis following resection of small hepatitis B-related hepatocellular carcinoma lesions are associated with aberrant tumor expression profiles of glypican 3 and osteopontin. Ann Surg Oncol 2012; 19(Suppl 3): S455-S463.

26. Iqbal J, McRae S, Banaudha K, Mai T, Waris G. Mechanism of hepatitis C virus $(\mathrm{HCV})$-induced osteopontin and its role in epithelial to mesenchymal transition of hepatocytes. J Biol Chem 2013; 288: 36994-37009.

27. Hasenfuss SC, Bakiri L, Thomsen MK, Hamacher R, Wagner EF. Activator Protein 1 transcription factor Fos-related antigen 1 (Fra-1) is dispensable for murine liver fibrosis, but modulates xenobiotic metabolism. Hepatology 2014; 59: 261-273.

28. Eferl R, Hasselblatt $P$, Rath M, Popper H, Zenz R, Komnenovic V et al. Development of pulmonary fibrosis through a pathway involving the transcription factor Fra-2/AP-1. Proc Natl Acad Sci USA 2008; 105: 10525-10530.

29. Wang X, Lopategi A, Ge X, Lu Y, Kitamura N, Urtasun R et al. Osteopontin induces ductular reaction contributing to liver fibrosis. Gut 2014; 63: 1805-1818.

30. Lee JS, Heo J, Libbrecht L, Chu IS, Kaposi-Novak P, Calvisi DF et al. A novel prognostic subtype of human hepatocellular carcinoma derived from hepatic progenitor cells. Nat Med 2006; 12: 410-416.

31. Behrens A, Sibilia M, David JP, Mohle-Steinlein U, Tronche F, Schutz G et al. Impaired postnatal hepatocyte proliferation and liver regeneration in mice lacking $c-j u n$ in the liver. Embo J 2002; 21: 1782-1790.

32. Stross L, Gunther J, Gasteiger G, Asen T, Graf S, Aichler M et al. Foxp3+ regulatory $T$ cells protect the liver from immune damage and compromise virus control during acute experimental hepatitis B virus infection in mice. Hepatology 2012; 56: 873-883. 